\title{
Accelerated myocardial perfusion imaging using saturation-recovery spiral-in/out SSFP
}

\author{
Xue Feng ${ }^{1 *}$, Yang Yang ${ }^{1}$, Craig $\mathrm{H}$ Meyer ${ }^{1,2}$, Michael Salerno ${ }^{3,2}$ \\ From 18th Annual SCMR Scientific Sessions \\ Nice, France. 4-7 February 2015
}

\begin{abstract}
Background
Myocardial first-pass perfusion imaging is a promising diagnostic tool for the assessment of ischemic heart disease. Spiral imaging, due to its high acquisition speed and robustness against motion artifacts, has been successfully combined with the saturation recovery (SR) GRE sequence using a traditional spiral-out trajectory. However, a SR SSFP sequence can provide higher SNR and use shorter saturation recovery time. In addition, as compared to a spiral-out trajectory, the spiral-in/out trajectory in an SSFP sequence can take advantage of the refocusing mechanism at $\mathrm{TE}=\mathrm{TR} / 2$ to increase $\mathrm{SNR}$ and reduce off-resonance artifacts. Furthermore, spatial and temporal parallel imaging methods can be used to achieve up to $4 \mathrm{x}$ acceleration without degrading the image quality. In this study, we have implemented a spiral-in/out SR SSFP sequence and imaged rest myocardial perfusion.
\end{abstract}

\section{Methods}

In the spiral-in/out SR SSFP sequence, a $90^{\circ} \mathrm{SR}$ pulse is followed by a fat sat pulse and a $40 \mathrm{~ms}$ SR time. The fully sampled spiral-in/out trajectory was designed with 48 interleaves and 1.6 ms readouts with a linearly decreasing sampling density to increase acquisition efficiency. Since $4 \mathrm{x}$ undersampling is used, 12 equally spaced interleaves were acquired at each frame and rotated by $90^{\circ}$ for subsequent frames. To reduce the phase error, a series of 8 linearly ramping RF pulses with data acquisition starting after the first 4 pulses were applied. Two additional spiral interleaves were acquired for off-resonance field map correction. The total imaging time per slice was $110 \mathrm{~ms}$.
Three patient studies were performed on a Siemens Avanto 1.5T scanner with informed consent under the local IRB. Perfusion images with 340-400 mm FOV and 1.8-2.1 mm spatial resolution at 4-6 short axis slice locations were acquired after Gd injection during an expiratory breath-hold. Chest and spine coils with up to 18 channels were used to facilitate the spatial parallel imaging technique and improve SNR. The missing $\mathrm{k}$-space data was interpolated using SLAM and fed into the SPIRiT reconstruction method as an additional data constraint. The data fidelity parameters were chosen to be smaller for the interpolated k-space data than those of the acquired k-space data since the former is not as reliable as the latter.

\section{Results}

Fig. 1 shows a series of myocardial perfusion images acquired using the SR SSFP sequence at 4 short axis positions. Banding artifacts are minimized with the ramping RF pulses and short TR. The proposed image reconstruction method successfully eliminates the spatial aliasing with the undersampled trajectory. With the high spatial resolution $(1.8 \mathrm{~mm})$, the coronary arteries are visible on multiple slices.

\section{Conclusions}

We have implemented an accelerated SR SSFP sequence for myocardial first-pass perfusion imaging using the spiral-in/out trajectory. Due to its high SNR and resolution, it is a promising alternative to the traditional GRE sequence.

\section{Funding}

NIH R01HL079110, NIH K23HL112910-02 and Siemens Medical Solutions.

${ }^{1}$ Biomedical Engineering, University of Virginia, Zion Crossroads, VA, USA Full list of author information is available at the end of the article 

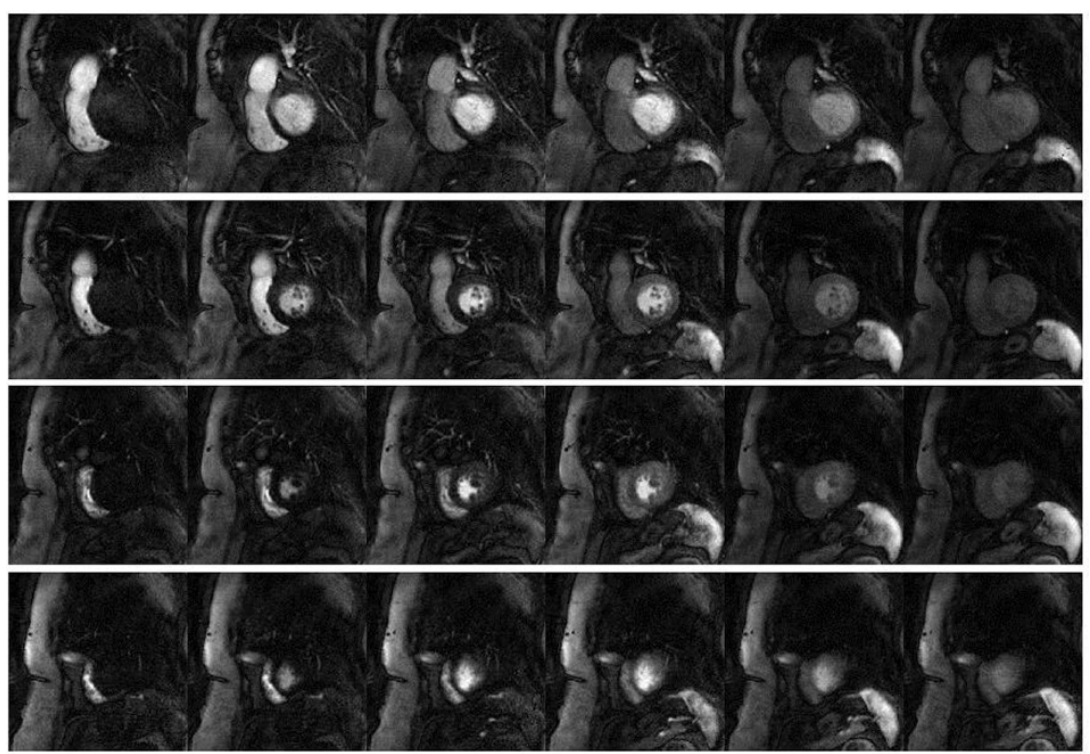

Figure 1 Myocardial perfusion images at every fourth frame acquired at 4 slice locations (row 1, 2, 3, 4) using the accelerated SR spiral-in/out SSFP sequence.

\section{Authors' details}

'Biomedical Engineering, University of Virginia, Zion Crossroads, VA, USA.

${ }^{2}$ Radiology, University of Virginia, Charlottesville, VA, USA. ${ }^{3}$ Medicine,

University of Virginia, Charlottesville, VA, USA.

Published: 3 February 2015

doi:10.1186/1532-429X-17-S1-P408

Cite this article as: Feng et al: Accelerated myocardial perfusion

imaging using saturation-recovery spiral-in/out SSFP. Journal of

Cardiovascular Magnetic Resonance 2015 17(Suppl 1):P408.

\section{Submit your next manuscript to BioMed Central} and take full advantage of:

- Convenient online submission

- Thorough peer review

- No space constraints or color figure charges

- Immediate publication on acceptance

- Inclusion in PubMed, CAS, Scopus and Google Scholar

- Research which is freely available for redistribution 